\title{
ANÁLISIS BIBLIOMÉTRICO SOBRE LA CREACIÓN DE EMPRESAS DE BASE TECNOLÓGICA DE ORIGEN UNIVERSITARIO
}

\section{Recepción: 8 de Septiembre del 2017; Aceptación: 6 de Octubre del 2017}

Publicación: 15 de Diciembre del 2017

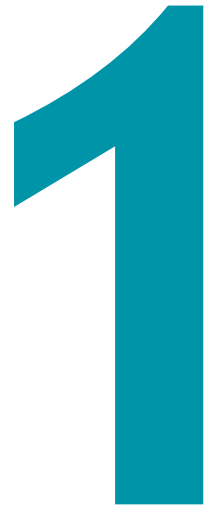

Julio César García Martínez ${ }^{1}$ Ma. Guadalupe Rivera Cano ${ }^{2}$ Abril Alejandra Ramírez Erazo ${ }^{3}$

\section{RESUMEN}

Se realiza un análisis bibliométrico de la literatura existente que ha tenido mayor impacto sobre el tema de creación de empresas de base tecnológica de origen universitario, el cual permite observar el desarrollo de la literatura enfocada en este tema de manera que a partir de ésta selección se pueda construir en posteriores estudios un marco teórico con base en una revisión sistemática y exhaustiva de la literatura. Siguiendo un diseño de tipo descriptivo/longitudinal, se pretende aportar información a la comunidad de investigadores en relación con la evolución y el impacto de los artículos publicados.

Para llevar a cabo la recolección de datos se utilizaron dos juegos de palabras, éstas se introdujeron en los motores de búsqueda de Science Direct, Emerald Insight, SpringerLink, Redalyc y Scopus, ya que significan bases de datos importantes para la búsqueda de investigaciones y artículos confiables en este tipo de temas. Los principales hallazgos obtenidos son que, el área donde más se desarrolla es en Negocios, Administración y Contabilidad.

El país con más publicaciones es Italia, la revista que más publica sobre este tema es Research Policy y una de las universidades más representativas es la de Gent (Bélgica). También se identificaron tres redes de colaboración, una de los cuales es liderada por Mike Wright que individualmente es el autor más productivo y con mayor impacto en el tema de las spin-off universitarias ${ }^{4}$.

Palabras clave: Análisis bibliométrico, empresas de base tecnológica, Spin Off Universitarias.

\section{ABSTRACT}

A bibliometric analysis of the existing literature with the most impact on the subject creation of technology based enterprises originated in universities is elaborated, in such a way that this selection can help build a theoretical framework based on a systematic and thorough literature review. Following a descriptive/transverse design, it is pretended to contribute with information to the community of researchers related to the evolution and impact of the pu- blished articles on this matter. In order to complete the data collection, two sets of words were used, these were entered in the search engine of the data bases: Science Direct, Emerald Insight, Springer Link, Redalyc and Scopus, since those are important sources of investigations and articles trustworthy on the subject. The main findings are that; the areas where ithe subject is mostly developed are Business, Administration and Accounting. The country with the most publications about the subject is Italy, the journal that publishes the most about it is Research Policy and one of the most representative universities is Gent (Belgium). Three collaboration networks were also identified, one of them led by Mike Wright, who individually is the most productive author and the one with the most impact on the subject of university spin-offs.

Keywords: Bibliometric analysis, Technology Based Enterprises, University Spin-Off.

E-mail:jgarciam@upv.edu.mx, teléfono: 8333340037, Universidad Politécnica de Victoria

E-mail:Iriverac16@gmail.com, celular: 8361041455 Universidad Politécnica de Victoria

${ }_{3}$ E-mail:aramireze@upv.edu.mx, teléfono: 8341264392, Universidad Politécnica de Victoria

${ }_{4}$ En el presente documento se utiliza el término de spin-off universitaria como sinónimo de empresa de base tecnológica de origen universitario. 


\section{DESCRIPCIÓN DEL PROBLEMA}

Numerosos estudios dan cuenta del papel que juegan las pequeñas empresas en la innovación, en particular las relacionadas con las nuevas tecnologías. Por ejemplo, Scherer (1983) y Schwalbach \& Zimmermann (1991) han encontraron que la propensión a patentar es menor en las grandes empresas que en las medianas. Mientras que (Acs \& Audretsch 1990,1998 y Acs Z. J., et al. 2005) han encontrado de manera reiterada que las pequeñas empresas generan más innovaciones por empleado que las grandes y esta relación mejora cuando las pequeñas empresas están vinculadas con la universidad (Link \& Rees, 1990).

La actividad académica de emprendimiento es una fuente vital de innovación, empleo y crecimiento económico (Szopa, Marek, \& Magdalena, 2015) y en este sentido desde mediados de los 90, las universidades y organizaciones de investigación han estado envueltas crecientemente en la transferencia de conocimientos y tecnología al sector productivo con la creación de spin-offs, sobre todo después del establecimiento de la Bayh-Dole Act (1980) en los Estados Unidos y la implementación de políticas de propiedad intelectual similares en otras partes del mundo (García Martínez J. C., 2014).

La creación de empresas desde las universidades no sólo es una vía de transferencia de tecnología, sino también de desarrollo económico regional, así no sólo crean productos o servicios innovadores, sino que contribuyen también a la productividad y creatividad (Szopa, Marek, \& Magdalena, 2015). Por ello cobra especial relevancia su estudio en un país como México, donde más del $90 \%$ de las empresas son micro y pequeñas con un bajo contenido tecnológico y bajo potencial de crecimiento, ya que la creación de empresas basadas en conocimientos científicos y tecnológicos desarrollados desde la universidad se convierte en un recurso estratégico para impulsar las tasas de crecimiento económico e innovación.
Sin embargo, debido al carácter relativamente reciente del fenómeno, no abundan los estudios empíricos sobre el tema (Rodeiro Pazos, Fernández López, Otero González, \& Rodríguez Sandiás, 2012) y se desconoce de forma precisa cuáles deben ser los mecanismos idóneos para el desarrollo de tales empresas.

Por lo anterior se llevó a cabo un análisis bibliométrico que antecede al desarrollo de un proyecto que tiene como fin la identificación de los factores que promueven la creación de este tipo de empresas, de esta manera el presente estudio permite observar el desarrollo de la literatura enfocada en la creación de empresas de base tecnológica de origen universitario y servir como referencia introductoria así como aproximación preliminar para los investigadores que busquen familiarizarse con la literatura de la creación de spin-offs universitarias. A partir de ésta selección se puede contestar a las preguntas: ¿En qué área de especialización se ha publicado más sobre el tema?, ¿Que tendencia presenta la evolución del tema a través del tiempo?, ¿Cuál es el top 10 de revistas que publican acerca de él?, ¿Cuál es el top 10 de los centros de investigación que estudian el tema?, ¿Cuál es el top 10 de los países más representativos en el estudio del tema? y ¿Qué clústeres de colaboración existen en este tema? Con las respuestas a los anteriores cuestionamientos se busca construir un marco teórico con base en una revisión sistemática y exhaustiva de la literatura.

\section{OBJETIVO GENERAL}

Realizar un análisis bibliométrico de la literatura existente que ha tenido mayor impacto sobre el tema de creación de empresas de base tecnológica de origen universitario. 


\section{BASES TEÓRICAS 3.1.Bibliometría}

La realización del análisis bibliométrico resulta importante ya que evalúa el surgimiento y desarrollo del conocimiento asociado con alguna rama de la ciencia por medio de artículos e informes presentados generalmente en revistas científicas, este tipo de análisis determina una serie de indicadores significativos para medir material bibliográfico (Albort-Morant, LealRodriguez, Fernández-Rodríguez, \& Ariza-Montes, 2017).

Porbibliometría podemos entender la subdisciplina de la cienciometría que proporciona información sobre los resultados del proceso investigador, su volumen, evolución, visibilidad y estructura dentro de un tema específico (Escorcia Otalora, 2008).

Para realizar el análisis bibliométrico nos apoyaremos de algunas de sus leyes como la ley de la productividad de los autores, la cual enuncia una relación cuantitativa entre los autores y las contribuciones producidas en un campo dado a lo largo de un periodo de tiempo. De esta producción sólo una parte de los autores son responsables de gran variedad de trabajos científicos. Y la ley de dispersión de la bibliografía científica (Ley de Bradford) que señala que no todas las publicaciones son igual de consultadas, sino que unas pocas acumulan la mayor parte del consumo, el cual puede medirse por el volumen de acceso a los documentos o a partir de las citas que cada uno reciben (Ardanuy, 2012).

Además de las leyes bibliométricos, la cienciometría cuenta con diversos indicadores para realizar el análisis bibliométrico, estos indicadores se pueden dividir en tres tipos: cantidad (miden productividad), calidad (miden impacto) y estructurales (miden conexiones) (Cadavid Higuita, Awad, \& Franco Cardona, 2012). Bajo estos aspectos podemos utilizar diversos indicadores bibliométricos como indicadores de calidad científica; número y distribución de publicaciones; productividad de los autores; colaboración en publicaciones; indicador de impacto de los trabajos y de las fuentes; e índice de inmediatez (González de Dios, 1997).
De los indicadores citados se describen en seguida los que serán utilizados en el presente análisis. Indicadores de la importancia científica

Número y distribución de publicaciones: consiste en el cómputo del número de publicaciones de determinados grupos, instituciones o países, así como su distribución, para el caso de las revistas; del análisis se deduce la distribución y dispersión de los trabajos (Sancho, 1990).

Productividad de los autores: La productividad de un autor se define como el logaritmo del número de sus trabajos publicados (Sancho, 1990).

Colaboración en las publicaciones (índice firmas/ trabajo): Este indicador arroja la apertura o aislamiento de las comunidades científicas, con él se pueden detectar grupos y redes de colaboración temática, y ejes de influencia entre comunidades científicas y regiones, siendo posible identificar también la colaboración interinstitucional (De Filippo \& Fernández, 2002).

\section{Indicadores de impacto científico}

Estos indicadores se apoyan en el supuesto de que los trabajos importantes son usualmente citados, mientras que los irrelevantes se ignoran.

Indicador del impacto de los trabajos: número de citas recibidas (procedentes de publicaciones posteriores).

Los indicadores, en términos generales, representan una medición agregada y compleja que permite describir o evaluar un fenómeno, su naturaleza, estado y evolución. Con los indicadores bibliométricos descritos se podrá determinar:

a) Crecimiento de un campo del saber.

b) Evolución cronológica de la producción científica.

c) Productividad de los autores o instituciones.

d) Colaboración entre científicos e instituciones.

e) Impacto o visibilidad de las publicaciones en la comunidad científica.

f) Dispersión de las publicaciones científicas por tipo de fuente. 


\subsection{Empresas de Base Tecnológica de Origen Universitario (EBT universitarias)}

Las EBT de origen universitario son nuevas empresas que dependen del licenciamiento o asignación de propiedad intelectual universitaria para su formación (Wright, Clarysse, Lockett, \& Knockaert, 2008). Generalmente son fundadas por académicos o alumnos involucrados en la investigación que dio como resultado la propiedad intelectual que se pretende comercializar. Entre los factores que influyen en su creación destacan el nivel educativo de los emprendedores, sus experiencias previas con el sector industrial y las características de los entornos universitario e institucional.

\subsection{Factores que influyen en la creación de empresas de base tecnológica de origen universitario}

A continuación, se enlistan los factores que influyen en la creación de EBT universitarias; dichos factores se monitorearán en la literatura que arroje el análisis bibliométrico.

Tabla 1 Factores determinantes en la creación de EBT universitarias

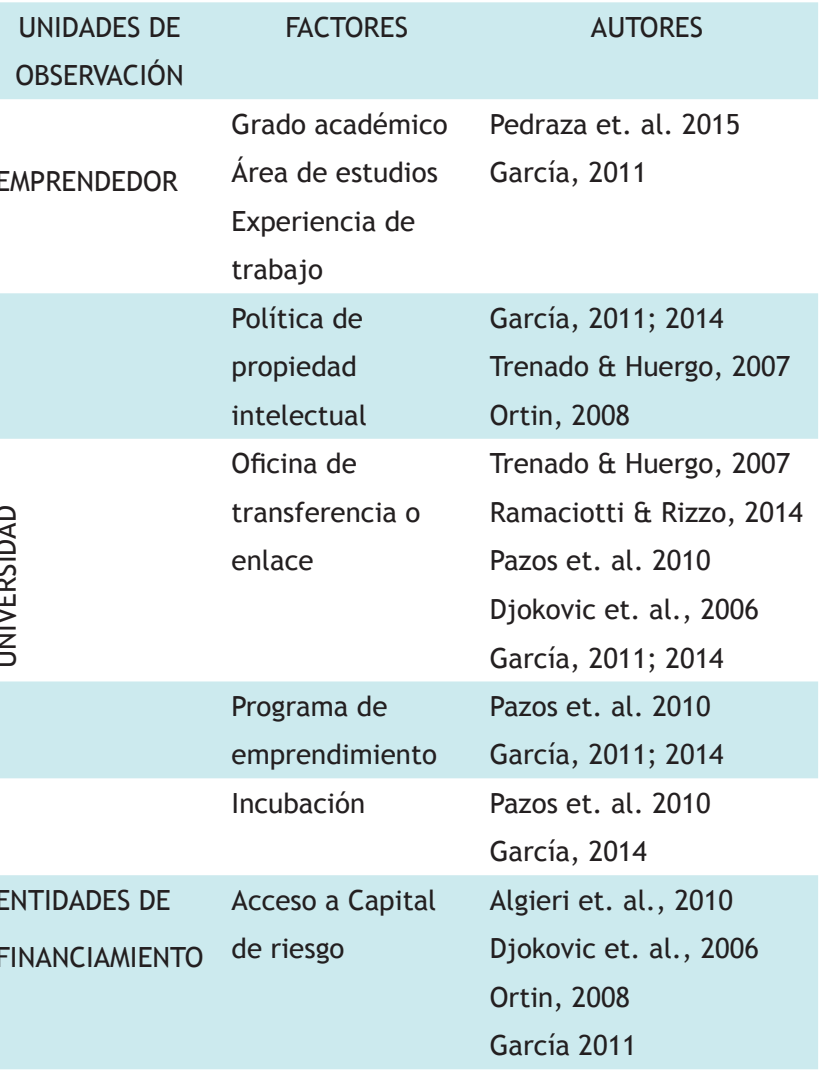

Fuente: modificado de (García Martínez J. C., 2011; 2014)

\section{METODOLOGÍA EMPLEADA}

Esta investigación es del tipo descriptivo ya que consiste en revisar, identificar y ordenar la información recabada a través de la lectura de los trabajos publicados de distintos autores en diferentes bases de datos en línea; y longitudinal ya que presenta la evolución de la producción científica a lo largo del periodo estudiado (Hernández Sampieri, Fernández Collado, \& Baptista Lucio, 2010).

La selección de variables se realizó conforme a Scopus, una de las bases de datos más importantes para la búsqueda de artículos de investigación, analizándose los siguientes indicadores bibliométricos:

a)Segmentación de áreas de especialización. Contiene los porcentajes con los que cuenta cada área para percibir en cuál de estas el tema a tratar ha sido más desarrollado.

b)Evolución del tema. A partir de una gráfica que representa una línea de tiempo se muestran los años y documentos registrados por año. Una línea de tendencia permite observar la evolución del tema desde la publicación del primer artículo.

c) Top 10 de revistas. Se refiere las revistas que más publican artículos del tema.

d)Top 10 de centros de investigación. Son las 10 instituciones que más investigaciones han realizado sobre el tema.

e)Top 10 de países representativos. En donde el tema ha tenido más presencia.

f)Clústeres de colaboración. Se muestran las redes de colaboración que existen entre autores. Se utilizaron los datos de Scopus para identificar los clústeres.

Para la recolección de datos se utilizaron dos juegos de palabras clave: "Create University Spin Off" (Creación de Spin Off Universitarias) y "Determinants of University Spin Off" (Determinantes en la creación de Spin Off Universitarias), estas se introdujeron en los motores de búsqueda de Science Direct, Emerald Insight, SpringerLink, Redalyc y Scopus. Utilizando a Scopus como la principal fuente para la recaudación completa de los artículos ya que brinda la facilidad de analizar los resultados de la búsqueda. 
La población está delimitada por artículos que hacen referencia a la Creación de Spin Off Universitarias y los Determinantes para la Creación de Spin Off Universitarias; por consiguiente, la muestra específica se lleva a cabo por filtro de los principales artículos que tratan de estos temas obteniendo como resultado 52 documentos.

\section{RESULTADOS}

Por medio de la utilización del análisis bibliométrico se dará luz en el tema de historia de esta ciencia, así como identificación de los países, instituciones y autores más productivos en el período de vida del tema, siendo estos algunos de los campos de aplicación más frecuente de este tipo de estudios (Araújo Ruiz \& Arencibia Jorge, 2002).

La base de datos de Scopus dividió los 52 resultados de la lista realizada a partir de la búsqueda de artículos en su motor. El primer indicador bibliométrico, es la segmentación de áreas de especialización en que se dividen los artículos encontrados. El área con más resultados fue Negocios, Administración y Contabilidad; seguida del área de Economía, Econometría y Finanzas; y de la de Ingeniería, siendo estas tres las que más se relacionan con el tema de las EBT. Tal y como se muestra en la gráfica 1 .

\section{Gráfica 1 Segmentación de áreas de especialización}

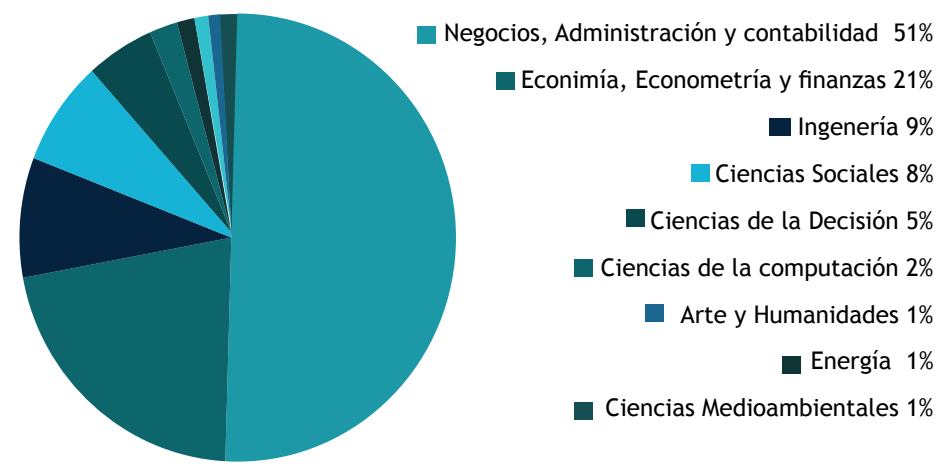

Fuente: elaboración a partir de Scopus.

En cuanto a la evolución se encontró que las publicaciones sobre el tema iniciaron en 1983 y con el paso de los años se ha incrementado el número de artículos. En los años 2014 y 2016 se observa la mayor producción de artículos llegando a 10 por año; en estos años se puede entender que el tema ha tenido mayor relevancia en el mundo.
Gráfica 2 Evolución del tema

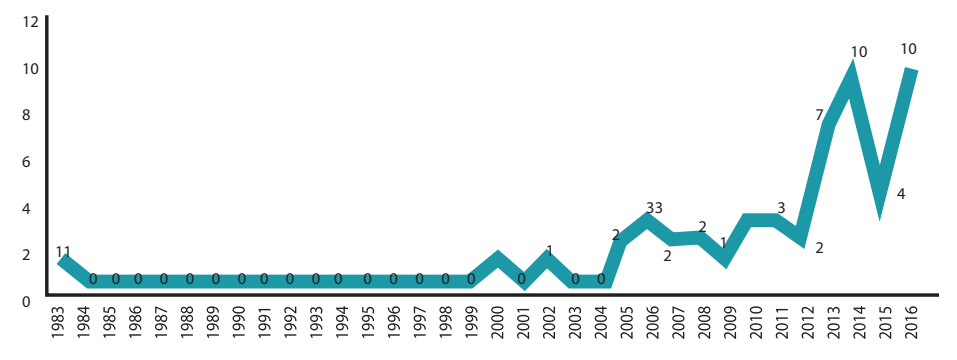

Fuente: elaboración a partir de Scopus.

El punto principal de los indicadores restantes es conocer la dispersión de las publicaciones por tipo de fuente. A continuación, se muestra la gráfica del indicador en dónde se muestra el top 10 de revistas más representativas del tema.

\section{Gráfica 3 Top 10 de revistas}

Research Policy

International Entrepreneurship Journal of Technology Transfer

Journal of International Entrepreneurship

Small Business Economics

Academia

Baltic Journal Of Management

California Management Review

Economic Development Quarterly

Economics of Innovation and New Technology

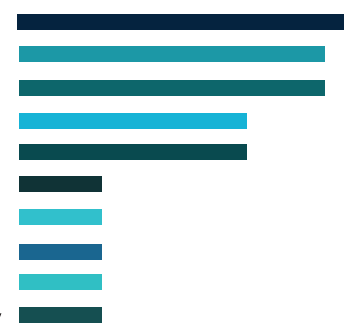

Fuente: elaboración a partir de Scopus.

De acuerdo a los resultados la revista que tiene más ediciones sobre el tema es "Research Policy" con 6 artículos publicados en sus ediciones hasta la actualidad.

En la siguiente gráfica se muestran los 10 principales centros de investigación que han realizado publicaciones sobre el tema.

\section{Gráfica 4 Top 10 de Centros de Investigación}

Universidad de Santiago de Compostela Universiteit Gent

Imperial College London

ALma Mater Studiorum Universita de Bologna

Universidade de Beira Interior

Universidade de Porto

National Univerity of Ireland Galway

University of Nottingham

Universidade de Coimbra

Univeridade de Coruña

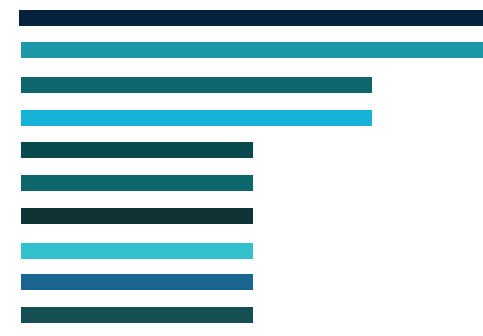

Fuente: elaboración a partir de Scopus.

En primer lugar, se muestra la Universidad de Santiago de Compostela en España, con un total de 4 documentos publicados al igual que la Universidad de Gante en Bélgica. 
Por último, el indicador de los países más representativos en donde se desarrollan dichos documentos se ilustra con el siguieente gráfico que contiene los 10 países que más presencia tienen, en él resulta evidente que el país que más produce esta clase de documentos es Italia con 13 artículos.

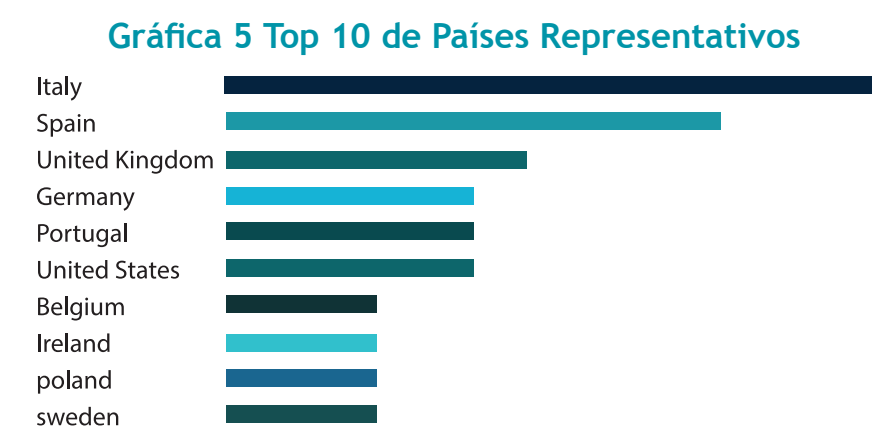

Fuente: elaboración a partir de Scopus.

A partir de los resultados obtenidos dentro de este análisis se elabora un diagrama de vectores utilizando al autor de cada artículo. Este diagrama permite determinar las relaciones existentes entre trabajos de investigación mediante la identificación de los autores con el mayor número de trabajos de investigación (García Martínez, Vasconcelo Álvarez, \& García Amado, 2015). Para su elaboración se hace uso de diferentes programas como lo son EndNote recopilador de citas bibliográficas, BibExcel que encuentra las relaciones de los autores de artículos científicos que se han recopilado con la búsqueda de los mismos y Pajek que ayuda a la visualización de este procedimiento. Una vez realizado el procedimiento anterior el resultado se muestra en la figura 1.

La formación de las redes de colaboración se considera a partir de la unión de diferentes trabajos de investigación, relacionados por los autores en común. (García et. al., 2015). Como se puede observar en la figura 1, en el tema de transferencia de conocimientos y spin-off universitarias existen tres redes principales lideradas por Arnaldo Coelho, Sara Fernández López y Mike T. Wright.

Figura 1 Redes de Investigación sobre las EBT de Origen Universitario

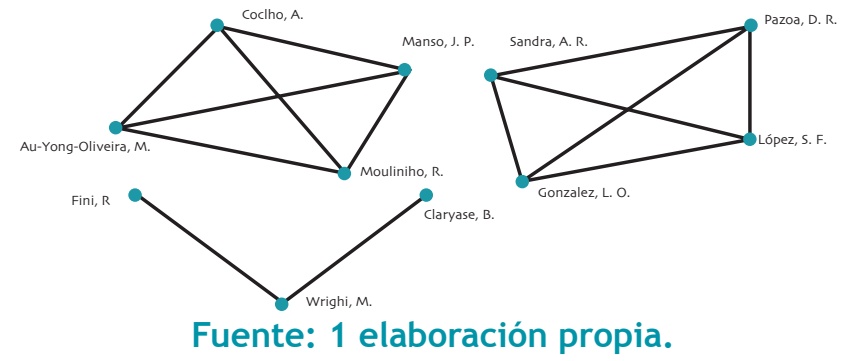

Para detallar de mejor manera el diagrama de vectores resultante Tang y Musa (2011) y Fahimnia et al. (2015) recomiendan el análisis de citación que estudia el nivel de impacto de las investigaciones en las disciplinas de estudio (García Martínez, Vasconcelo Alvarez, \& García Amado, 2015). En la tabla 2 se desarrolla el análisis de impacto que ha tenido cada autor en el tema de transferencia de conocimientos universidad-empresa y empresas de base científica/tecnológica dentro de cada red. El nivel de impacto del autor se basa en las citas en revistas indexadas en relación al número de publicaciones del autor a través del tiempo (García Martínez et. al., 2015). Dichos niveles e información son de la vida editorial de cada autor extraidos de la base de datos Scopus y no está limitada el tema específico que aquí nos ocupa.

Tabla 2 Citas y Nivel de impacto de autores

\begin{tabular}{cccc} 
CLÚSTER & AUTORES & CITAS & $\begin{array}{c}\text { IMPACTO } \\
\text { (H-INDEX) }\end{array}$ \\
\hline & COELHO, A. & 2 & 4 \\
MOURINHO, R. & 3 & 1 \\
MANSO, J.P. & 3 & 1 \\
\hline AU-YONG-OLIVEIRA, M. & 3 & 1 \\
LÓPEZ, S.F. & 18 & 2 \\
GONZÁLES, A, R. & 11 & 2 \\
SANDIÁS, A.R. & 9 & 2 \\
PAZOS, D.R & 7 & 1 \\
WRIGH, M. & 15679 & 68 \\
CLARYSSE, B. & 2359 & 25 \\
FINI, R. & 400 & 7
\end{tabular}

Fuente: 2 Elaboración a partir de Scopus.

En la tabla anterior se puede apreciar los autores con mayor productividad y su impacto a través de los trabajos que han sido citados, mediante el índice $\mathrm{H}$ (h-index) se ha medido el impacto que han tenido los autores hasta la actualidad. Por ejemplo, el índice $\mathrm{H}$ de Wright, $M$. es de 68, o sea, al ordenar los artículos que ha desarrollado en su vida editorial resultarán que el artículo número 68 se ha citado por lo menos 68 veces en distintos trabajos. De manera matemática se entenderá que un investigador tiene un índice $h$, si el $h$ de sus $\mathrm{N}$ trabajos recibe al menos $\mathrm{h}$ citas cada uno, y los otros (Np - h) trabajos tienen como máximo h citas cada uno. 


\section{CONCLUSIONES}

Se encontró que las principales áreas donde se desarrolla el tema de la creación de spin-offs universitarias es en Negocios, Administración y Contabilidad; Economía, Econometría y Finanzas; e Ingeniería, como era de esperarse. En cuanto a su evolución el tema todavía es joven, pero se encuentra en crecimiento, pues desde el año 1983 (año en que se publicó el primer artículo) hasta la actualidad se han publicado 52 artículos, sin embargo, es de destacar que estos guardan poca relación entre sí evidenciando un interés desagregado en el tema.

El análisis de las publicaciones realizadas durante 33 años, identifica los años con mayor actividad, los cuales podrían dividirse en tres etapas, la primera en la que aparece el tema por primera vez y muestra una producción baja de entre 0 y 1 por año en el periodo de 1983 a 2004, la segunda de 2005 a 2012 con entre 1 y 3 publicaciones por año y una tercera que presenta un mayor auge a partir del año 2013 en la cual se observa un aumento significativo con hasta 10 publicaciones por año sobre el tema, resultando ser a partir de este periodo cuando el tema presenta un mayor impacto, evidenciando el creciente interés en él por parte de la comunidad de investigadores en años recientes.

Research Policy es la principal revista de investigación que hace posible que se den a conocer publicaciones relativas al tema, concordando con los fines que ésta busca, pues su objetivo es publicar contenido que pueda llevar a hallazgos que tengan implicaciones respecto a políticas o gestión (Elsevier, s.f.). Entre las instituciones que más promueven el tema se encuentra la Universidad de Santiago de Compostela en España. Por otra parte, respecto a los países en los que se encuentra una mayor cantidad de publicaciones sobre el tema, resulta de gran interés señalar que el top 10 está conformado en su totalidad por países desarrollados, incluso cuatro de ellos pertenecientes al grupo de los 7 (G7), lo cual deja en evidencia la novedad aun mayor del tema en países menos desarrollados como es el caso de México, y para los cuales, la transferencia de tecnología juega un rol determinante en su desarrollo tecnológico.
También se trazó una red de coautores, obteniéndose como resultado que los autores que más colaboran entre sí son los que integran las redes lideradas por Arnaldo Coelho; Sara Fernández López; y Mike T. Wright; destacándose éste último por el nivel de productividad e influencia.

La creación de empresas de base tecnológica de origen universitario, es un tema que ha ido tomando fuerza en años recientes debido al desarrollo de nuevas plataformas tecnológicas y a la preocupación de los gobiernos por acelerar las tasas de innovación y el crecimiento económico a través del emprendimiento tecnológico, toda vez que la innovación tecnológica, incluyendo el desarrollo de nuevas fuentes de oferta constituye una de las principales fuerzas motrices del crecimiento económico y bienestar de la sociedad moderna (CONACYT). Sin embargo, los países de menor desarrollo aún se encuentran lejos de los primeros lugares en la producción del tema a pesar del alto potencial que tienen las nuevas tecnologías en generar nuevas industrias y empresas de rápido crecimiento debido a la ausencia de grandes competidores, incluso aun considerando que cerca del $50 \%$ de las empresas más dinámicas de América Latina han sido spin-offs universitarias (Parra Bernal \& Argote Cusi, 2015).

Los indicadores cienciométricos son utilizados frecuentemente en países desarrollados para definir políticas de apoyo a las actividades científicas y tecnológicas, de esta manera dichos países pueden conocer las áreas o especialidades, así como las instituciones que han logrado un mejor desempeño en determinado periodo de tiempo (Pérez Angón, 2006). Así mismo el presente estudio permite ubicar dónde se encuentra la frontera de conocimiento al identificar tanto los principales autores que han escrito sobre el tema como las principales universidades o centros de investigación que lo han estudiado. 


\section{BIBLIOGRAFÍA}

Acs, Z. J., \& Audretsch, D. B. (1990). Innovation and small firms. Cambridge: MIT Press, https://doi.org/10.1007/978-94-015-7854-7

Acs, Z. J., \& Audretsch, D. B. (1998). Innovation in Large and Small Firms: An Empirical Analisys. American Economic Review, 678-690.

Acs, Z. J., Audretsch, D. B., Braunerhjelm, P., \& Carlsson, B. (2005). The Knowledge Spillover Theory of Entrepreneurship. Entrepreneurship, Growth and Public Policy, Jena.

Albort-Morant, G., Leal-Rodriguez, A., Fernández-Rodríguez, V., \& Ariza-Montes, A. (2017). Assessing the Origins, Evolution and Prospects of the Literature on Dynamic Capabilities: A Bibliometric Analysis. European Research on Management and Business Economics, https://doi.org/10.1016/j.iedeen.2017.06.004

Araújo Ruiz, J., \& Arencibia Jorge, R. (2002). Informetría, bibliometría y cienciometría: aspectos teórico-prácticos. Acimed. Revista Cubana de los Profesionales de la Información y la Comunicación en Salud. Cuban Journal of Health Information and Communication Professionals, 10(4).

Ardanuy, J. (2012). Breve introducción a la bibliometría. España: Universitad de Barcelona.

Beraza Garmendia, J., \& Rodríguez Castellanos, A. (2012). Coceptualizacion de la Spin-Off Universitaria Revisión de la Literatura. País Vasco: Universidad del País Vasco.

Cadavid Higuita, L., Awad, G., \& Franco Cardona, C. (2012). Análisis Bibliométrico del Campo modelado de difusión de Innovaciones. Estudios Gerenciales, 28, 213-236, https://doi.org/10.18046/j.estger.2012.1486 CONACYT. (s.f.). Portal del Consejo Nacional de Ciencia y Tecnología. Recuperado el 10 de Agosto de 2017, de Información de Ciencia y Tecnología - Estudios de Ciencia y Tecnología: http://2006-2012.conacyt.gob.mx/ InformacionCienciayTecnologia/Paginas/

SitiosDeInteres.aspx\#EstudiosCiencia

De Filippo, D., \& Fernández, M. (2002). Bibliometría: importancia de los indicadores bibliométricos. El estado de la ciencia: principales indicadores deficiencia y tecnología Iberoamericanos/interamericanos.

Elsevier. (s.f.). Elsevier. Obtenido de https://www.journals.elsevier.com/research-policy Escorcia Otalora, T. A. (2008). El análisis bibliométrico como herramienta para el seguimiento de publicaciones centíficas, tesis y trabajos de campo. Bogotá: Pontificia Universidad Javeriana. García Martínez, J. C. (2011). Factores que influyen en la transferencia de conocimientos a través de incubadoras de empresas. Dos casos de estudio. México:

FLACSO-MÉXICO.
García Martínez, J. C. (2014). El entorno universitario como factor en la transferencia de conocimientos a través de incubadoras de empresas. Entreciencias. Diálogos en la sociedad del conocimiento, 227-244, https://doi.org/10.21933/J.EDSC.2014.05.013

García Martínez, J., Vasconcelo Álvarez, R., \& García Amado, J. (2015). Análisis bibliométrico de los modelos de negocios innovadores en la sustentabilidad. México: $4^{\circ}$ Coloquio de Investigación. Universidad Autónoma de Tamaulipas. González de Dios, J. (1997). Indicadores bibliométricos: Características y limitaciones en el análisis de la actividad científica. ANALES ESPAÑOLES DE PEDIATRIA, 47(3), 235244.

Hernández Sampieri, R., Fernández Collado, C., \& Baptista Lucio, M. (2010). Metodología de la Investigación (Quinta ed.). Méxigo D.F.: McGraw-Hill / Interamericana Editores.

Link, A. N., \& Rees, J. (1990). Firm Size, University Based Research and the Returns to R\&D. Small Business Economics, 25-32, https://doi.org/10.1007/BF00389891

Parra Bernal, L., \& Argote Cusi, M. (2015). Una Mirada a las Empresas de los Estudiantes y Egresados: El Caso de la Universidad EAN. Estudios Gerenciales, 122-134, https://doi.org/10.1016/j.estger.2014.06.008

Pérez Angón, M. (2006). Usos y abusos de la cienciometría. Cinvestav.

Rodeiro Pazos, D., Fernández López, S., Otero González, L., \& Rodríguez Sandiás, A. (2012). A resource -based view of university spin-off activity: new evidence from the spanish case. España: Elsvier, https://doi.org/10.1016/j.redee.2012.05.006 Sancho, R. (1990). Indicadores Bibliométricos Utilizados en la Evaluación de la Ciencia y la Tecnología. Revisión Bibliográfica. Revista Española de Documentación Científica, 842-865, https://doi.org/10.3989/redc.1990.v13.i3.842

Scherer, F. M. (1983). "The propensity to patent". International Journal of Industrial Organization, 107-128, https://doi.org/10.1016/0167-7187(83)90026-7

Schwalbach, J., \& Zimmermann, K. F. (1991). A poison model of patenting and firm structure in Germany. En Z. J. Acs, \& D. B. Audretsch, Innovation and Technological Change: An International Comparison (págs. 109-120). Michigan: University Michigan Press.

Szopa, A., Marek, T., \& Magdalena, F. (2015). Socio-Cultural Circumstances to Establish University Spin-Off Companies.

Procedia Manufacturing, 3, 3677-3687, https://doi.org/10.1016/j.promfg.2015.07.780

Wright, M., Clarysse, B., Lockett, A., \& Knockaert, M. (2008). Mid-range universities' linkages with industry: Knowledge types and role of intermediaries. Research Policy, 37(8), 1205-1223, https://doi.org/10.1016/j.respol.2008.04.021 
Copyright (c) 2017 Julio César García Martínez, Guadalupe Rivera Cano \& Abril Alejandra Ramírez Erazo

\section{(1) $\Theta \Theta$}

Este texto está protegido por una licencia Creative Commons 4.0.

Usted es libre para Compartir (copiar y redistribuir el material en cualquier medio o formato), siempre que cumpla las condiciones de:

Atribución: Usted debe dar crédito a la obra original de manera adecuada, proporcionar un enlace a la licencia, e in dicar si se han realizado cambios. Puede hacerlo en cualquier forma razonable, pero no de forma tal que sugiera que tiene el apoyo del licenciante o lo recibe por el uso que hace de la obra.

No Comercial: Usted no puede hacer uso de la obra con propósitos comerciales

Sin Derivadas: Si remezcla, transforma o crea a partir de la obra, no podrá distribuir la obra modificada

$\underline{\text { Resumen de la licencia - Texto completo de la licencia }}$ 